\title{
SGLT2 Inhibitors Through the Windows of EMPA-REG and CANVAS Trials: A Review
}

Ashu Rastogi · Anil Bhansali

Received: September 17, 2017 / Published online: October 26, 2017

(C) The Author(s) 2017. This article is an open access publication

\begin{abstract}
EMPA-REG OUTCOME and CANVAS trials were designed to study the cardiovascular safety of empagliflozin and canagliflozin, respectively. Both studies were sufficiently powered to study the non-inferiority for cardiovascular (CV) outcomes in patients with type 2 diabetes mellitus (DM) and showed superiority for major adverse cardiovascular events and composite renal outcomes independent of glycemic control. Further, all patients in EMPA-REG had prior CV events (secondary prevention), compared to CANVAS that also included subjects with no prior CV events, indicating the beneficial effects of canagliflozin in primary prevention of $\mathrm{CV}$ events as well. Moreover, there seems to be ethnic variations in response to sodium-glucose cotransporter 2 inhibitors (SGLT2i) regarding $\mathrm{CV}$ benefits, as Blacks fared better with canagliflozin and Asians with empagliflozin. Increases in lower extremity amputation and fracture incidence were observed with canagliflozin in CANVAS and this needs further substantiation,
\end{abstract}

Enhanced content To view enhanced content go to http://www.medengine.com/Redeem/ 04CCF06050F44F7F.

\section{A. Rastogi · A. Bhansali ( $\square)$}

Department of Endocrinology, PGIMER, Chandigarh 160012, India

e-mail: anilbhansaliendocrine@gmail.com though these events were not systematically captured in the EMPA-REG study.

Keywords: Cardiovascular outcome trials; Cardiovascular mortality; Canagliflozin; CANVAS; Diabetes mellitus; Empagliflozin; EMPA-REG; Hazard ratio (HR); Major adverse cardiovascular events (MACE); Sodium-glucose linked cotransporter inhibitors (SGLT2i)

\section{INTRODUCTION}

The US Food and Drug Administration (FDA) 2008 regulations mandate the sponsors to conduct cardiovascular outcome trials (CVOTs) for ensuring cardiovascular (CV) safety of newly marketed antidiabetes agents [1]. The sodium-glucose cotransporter inhibitors (SGLT2i) have entered the world market after this mandatory requirement, hence necessitating the need for CVOTs. These trials are required to exhibit a two-sided 95\% CI upper boundary of hazard ratio (HR) of less than 1.3 for major adverse cardiovascular events (MACE) in comparison to the control group $[1,2]$. Therefore, CVOTs have been conducted for both empagliflozin and canagliflozin to test for non-inferiority for the prespecified primary $\mathrm{CV}$ outcome variables with HR of less than 1.3. Understandably, the population included in these CVOTs had high CV risk, so as to have 
maximum CV events (CVE) within the stipulated time period, thus providing an opportunity to assess the CV safety of these drugs [2-4].

The CV outcome results of empagliflozin cardiovascular outcomes and mortality in type 2 diabetes trial (EMPA-REG) [3] and canagliflozin cardiovascular assessment study (CANVAS) [4] with empagliflozin and canagliflozin, respectively, have demonstrated tangible benefits beyond the thoughts of the diabetes-treating community. Previously, metformin and liraglutide have demonstrated CV benefits independent of glycemic control in patients with T2DM in the UKPDS study and LEADER study, respectively $[5,6]$. The SGLT2i are another class of oral antihyperglycemic drugs (OADs) that have exhibited beneficial CV outcomes independent of glycemic control. Do the $\mathrm{CV}$ benefits with empagliflozin and canagliflozin mirror each other and have similar implications? We herein dissect the CV and renal outcomes of EMPA-REG and CANVAS trials with their implications in clinical practice.

This article is based on previously conducted studies and does not involve any new studies of human or animal subjects performed by any of the authors.

\section{STUDY DESIGN}

Both CANVAS and EMPA-REG trials had a large sample size, included subjects with high CV risk, had well-defined primary endpoints and predefined criteria for event adjudication. All patients in EMPA-REG study had prior CV events compared to CANVAS that also included subjects (33\%) with no prior CV events. However, CANVAS is an analysis of pooled results of two separate cohorts (CANVAS and CANVAS-R) followed for different durations. Projected median follow-up of CANVAS and CANVAS-R was 5.69 and 2.04 years, respectively, with follow-up duration of 2.42 years in the integrated CANVAS program, while EMPA-REG had a follow-up of 3.01 years $[3,7,8]$. Both these trials were of sufficient duration and satisfied the FDA criteria for long-term ( $>2$ years) studies to assess the effects of the investigational drug on $\mathrm{CV}$ outcomes. The CANVAS trial included the pooled results of two separate cohorts, i.e., CANVAS primarily aimed for three-point major adverse cardiovascular events (3P-MACE) and CANVAS-R for renal outcomes, which is likely to introduce an inherent bias for the analysis of primary outcomes, as pooled data of two different cohorts with separate aims makes it statistically less robust. Nevertheless, the FDA allowed pooling of the results, as these trials were event-driven [8].

Moreover, there was a wide ethnic disparity in the enrollment of patients, as CANVAS and EMPA-REG had only $13 \%$ and $21 \%$ of subjects from Asia, respectively, despite Asians accounting for almost half of the world's diabetic population $[3,7,9]$. Conventionally, the CVOTs are designed to analyze the prespecified primary endpoints; in addition, a few exploratory variables like hospitalization for heart failure, all cause mortality, etc., are also studied, as they are patient-important events and provide additional information beyond the primary outcome variables. But, the statistical analysis for these exploratory variables may not be so robust as the study may be underpowered for these outcome measures and may attenuate the significance of the results. However, both the EMPA-REG and CANVAS trials examined the exploratory variables despite this limitation $[3,4,8]$.

\section{OUTCOME}

Canagliflozin decreased the 3P-MACE by $14 \%$ (HR 0.86, 95\% CI 0.75-0.97), which is a composite of $\mathrm{CV}$ death, non-fatal myocardial infarction and non-fatal stroke in CANVAS, identical to empagliflozin (HR 0.86; 95\% CI $0.74-0.99, p=0.04$ for superiority) in the EMPA-REG study $[3,4]$. Both these SGLT2i have demonstrated superiority over the placebo with regards to the primary outcome.

The individual components of 3P-MACE in the EMPA-REG trial showed a trend for non-inferiority (HR either side of 1.0), except for CV deaths (HR 0.62, 95\% CI 0.49-0.77), which was the major driver for 3P-MACE reduction. One can thereby deduce that empagliflozin reduces the likelihood of $\mathrm{CV}$ death 
without significantly reducing the CV events, which is ironic, as the drug seems not to prevent the illness but the death. This can be explained as $40 \%$ of deaths in the EMPA-REG trial were due to "undefined causes", which were incriminated as CV deaths. After elimination of these "non-assessable" deaths from the analysis, the superiority of empagliflozin was abrogated (HR 0.90, 95\% CI 0.77-1.06) [10]. All the individual components of MACE showed non-inferiority with canagliflozin (95\% CI of HR on either side of 1.0), while the composite primary outcome of 3P-MACE showed a trend for superiority. What drives the superiority of canagliflozin over placebo for 3P-MACE outcome is difficult to understand, unlike empagliflozin.

A profound reduction (38\%) in CV death was observed with empagliflozin, compared to only $13 \%$ reduction in CV death with canagliflozin on top of optimal secondary preventive measures including aspirin and statin therapy, despite being from the same class. This could be related to the differences in the baseline characteristics of the study population, effect of concurrent use of cardioprotective drugs, selectivity for SGLT2 over SGLT1 transporters (>2500-fold for empagliflozin and >250-fold for canagliflozin) [11, 12], adjudication of CV events or statistical analysis of the results. The differences in the baseline characteristics (Table 1) of the study subjects might explain the disparity in observed CV outcomes [3, 4, 7]. Two-thirds of patients in the CANVAS trial had established CV events and one-third were at risk for CV events, whereas EMPA-REG enrolled $99.5 \%$ of subjects with prior CV events. It is presumed that the higher the baseline HbA1c is, the greater the HbA1c reduction with any intervention will be. In a similar way, the higher the baseline risk for $\mathrm{CV}$ events is, the better the $\mathrm{CV}$ protection will be, as was shown in the EMPA-REG trial. Is it so simple to confirm this presumption? Yes, it is probably true, as subjects with prior $\mathrm{CV}$ events in the CANVAS trial had $18 \%$ reduction in CV death (HR 0.82, 95\% CI $0.72-0.95)$ compared to only $2 \%$ in those without prior CV events (HR 0.98, 95\% CI 0.74-1.30). Further, the follow-up duration could have influenced the MACE outcome in the study groups, as duration of study was
Table 1 Baseline characteristics of included subjects in two trials

\begin{tabular}{|c|c|c|}
\hline Parameters & EMPA-REG & CANVAS \\
\hline Number of subjects & 7020 & 10,142 \\
\hline Age (years) & 63.2 & 63.3 \\
\hline $\begin{array}{l}\text { Duration of diabetes } \\
\text { (years) }\end{array}$ & - & 13.5 \\
\hline BMI $\left(\mathrm{kg} / \mathrm{m}^{2}\right)$ & 30.5 & 32 \\
\hline HbAlc (baseline) (\%) & 8.0 & 8.2 \\
\hline Prior CVD (\%) & 99.5 & 65.6 \\
\hline $\begin{array}{l}\text { Duration of follow-up } \\
\text { (years) }\end{array}$ & 3.1 & $\begin{array}{c}6.0 \text { (CANVAS } \\
\text { R, } 2 \text { year) }\end{array}$ \\
\hline eGFR $\left(\mathrm{ml} / \mathrm{min} / 1.73 \mathrm{~m}^{2}\right)$ & 83.1 & 76.5 \\
\hline $\begin{array}{l}\text { eGFR }<60 \mathrm{ml} / \mathrm{min} / \\
1.73 \mathrm{~m}^{2}(\% \text { of subjects })\end{array}$ & 26.0 & - \\
\hline
\end{tabular}

$B M I$ body mass index, $C V D$ cardiovascular disease, $e G F R$ estimated glomerular filtration rate

double with CANVAS compared to EMPA-REG (6 vs 3 years).

The glycemic equipoise, defined as the difference in HbA1c achieved between the drug and the placebo arm, should not exceed $0.3 \%$ at the end of the study, which is a prerequisite for CVOTs to abrogate the effect of reduction in glucotoxicity on CV outcomes [13]. The HbA1c differences in the drug arm versus placebo were $0.28 \%$ and $0.58 \%$, in EMPA-REG and CANVAS trials, respectively. Hence, some beneficial effects on CV outcomes with canagliflozin may be attributed to glycemic reduction unlike empagliflozin.

\section{TIME POINT FOR CV BENEFITS}

Divergence in the survival curve for MACE was observed at 3 months in the EMPA-REG study (as the curved lines started drifting at 3 months) as opposed to at approximately 1 year in the CANVAS trial $[3,4]$. An early separation in the survival curve lines with empagliflozin has been attributed to the hemodynamic effects [14], 
alternate fuel hypothesis $[15,16]$, favorable lipid profile [3], and improved endothelial function [3]. However, the effect of canagliflozin on MACE was observed after a year, thereby suggesting the predominant favorable effect on atherosclerosis, as the process of atherosclerosis is heralded only in months or years and not within a few weeks of intervention. However, all plausible explanations require further substantiation.

\section{ETHNIC VARIATIONS IN CV BENEFITS}

There were appreciable ethnic differences in the $\mathrm{CV}$ outcomes in both trials. Asian and Caucasians had better CV benefits than Blacks in the EMPA-REG study, whereas canagliflozin was superior in Blacks and Caucasians as compared to Asian subjects (Table 2) [3, 4]. This observation may be attributed to variations in racial response to treatment because of differential expression of SGLT2 in the kidney or variability in death adjudication at individual sites or may be unknown factors. Similar geographical variations in all-cause and CV mortality have been observed amongst patients with diabetes from North America and the rest of the world with intensive versus conventional glycemic control across ACCORD, ADVANCE, PROactive, UDGP, and VADT trials [17]. There was an increase in all-cause and CV mortality in North America compared with the rest of the world. However, none of the studies mentioned above including CANVAS and EMPA-REG were powered enough to assess the ethnic or geographical variations in outcome.

Table 2 Ethnic variation in effect size on MACE in EMPA-REG and CANVAS trials

\begin{tabular}{llllll}
\hline \multirow{2}{*}{ Ethnicity } & \multicolumn{2}{l}{ EMPA-REG } & & \multicolumn{2}{l}{ CANVAS } \\
\cline { 2 - 3 } & HR & CI & & HR & CI \\
\hline Caucasian & 0.88 & $0.74-1.04$ & & 0.84 & $0.73-0.96$ \\
Asian & 0.68 & $0.48-0.95$ & & 1.08 & $0.72-1.64$ \\
Black & 1.48 & $0.80-2.02$ & & 0.45 & $0.19-1.03$ \\
\hline
\end{tabular}

$H R$ hazard ratio, $C I$ confidence interval

\section{EFFECT ON NON-FATAL STROKE}

Canagliflozin reduced the risk of non-fatal stroke by 10\% (HR 0.90, 95\% CI 0.71-1.15), unlike empagliflozin that increased the risk by 24\% (HR 1.24, 95\% CI 0.92-1.67), though the difference was not statistically significant $(p=0.16)[3,4]$. Further, the hazard ratio for stroke was similar irrespective of systolic [HR $1.14(0.77,1.68 \mathrm{CI})]$ and diastolic blood pressure control [HR $1.23(0.82,1.85 \mathrm{CI})]$ (> 140/90 versus $<140 / 90 \mathrm{mmHg}, p=0.79$ for interaction) with empagliflozin in the EMPA-REG study [3]. A higher rate of stroke incidence observed with empagliflozin was in spite of a similar reduction in systolic and diastolic blood pressure with either empagliflozin or canagliflozin in the two trials $[3,4]$. However, the difference in non-fatal stroke outcomes between the two trials remains unexplained. It may be attributed to diuresis-induced dehydration and increased viscosity in EMPA-REG; but this seems unlikely, as the results were not replicated with canagliflozin despite increased adverse effect reporting of volume depletion with canagliflozin (HR 1.44, 95\% CI 1.09-1.90) [4]. In addition, inclusion of greater numbers of subjects with prior stroke in EMPA-REG than in the CANVAS trial $(23 \%$ vs $19.3 \%)$ could have led to this outcome $[3,4]$.

\section{EFFECT ON RENAL OUTCOME}

Both EMPA-REG [18] and CANVAS [4] trials demonstrated significant renal benefits despite renal outcome measures adjudicated in the two trials being broadly dissimilar and annihilating a head-to-head comparison of renal benefits. CANVAS participants had a $27 \%$ reduction in albuminuria progression (HR 0.73, 95\% CI $0.67-0.79)$ and $40 \%$ reduction in the composite renal outcome (HR 0.60, 95\% CI 0.47-0.77) [defined as $40 \%$ reduction in the estimated glomerular filtration rate (eGFR), the need for renal replacement therapy, or death from renal causes]. The EMPA-REG study also showed a $38 \%$ reduction (HR $0.62,95 \%$ CI $0.54-0.72$ ) in progression to macroalbuminuria and $39 \%$ reduction in incident or worsening nephropathy (HR 0.61, 95\% CI 0.53-0.70) (defined as 
progression to macroalbuminuria, doubling of serum creatinine, requirement for renal replacement therapy, or death due to renal events). Therefore, the two trials inadvertently had a different subset of outcome variables but both showed a favorable renal outcome at the end of the study. Renal benefits have been accredited to hemodynamic factors and restoration of tubuloglomerular feedback. Further, it has been shown that SGLT1 co-transporters are overexpressed in proximal convoluted tubules following the prolonged use of SGLT2i. It was assumed that canagliflozin might fare better in terms of renal outcomes than empagliflozin because of concurrent inhibition of SGLT1 (relative non-selectivity) co-transporters by canagliflozin but not by empagliflozin. However, the renal benefits were similar between the two studies.

\section{ADVERSE EVENTS}

The incidence rates of common adverse events, with the use of SGLT2i, namely genital mycotic infections and volume depletion, were similar with both drugs. One surprise was the almost twofold increased risk for lower extremity amputations (LEA) with canagliflozin (HR 1.97; 95\% CI 1.41-2.75, $p<0.001)$, unlike empagliflozin. Further, the percentage of subjects with prior amputation in two arms (canagliflozin and placebo arm) of CANVAS was similar. Amputations in CANVAS were observed more often in men, and in those with history of prior amputation, neuropathy or peripheral vascular disease [4]. Further the amputation risk was not different between 100 and $300 \mathrm{mg}$ doses of canagliflozin. The amputation events were not systematically captured in EMPA-REG; hence, it was not possible to extrapolate the same risk with empagliflozin. However, a total of 131 patients in EMPA-REG had LEA, but the incidence was similar between placebo and empagliflozin (0.2\% each) groups [3]. Further, the US FDA Adverse Event Reporting System (FAERS) analysis exhibited a higher frequency of LEA (including any amputation and toe amputation) with canagliflozin with a proportional reporting ratio (PRR) of 5.33 (95\% CI 4.04-7.04, $p<0.0001), 0.25$ with dapagliflozin, and 2.37 with empagliflozin compared to non-SGLT2i agents for diabetes indication [19]. The risk of LEA with canagliflozin was more compared to with non-SGLT2i, despite that many patients with non-SGLT2i were receiving insulin, a marker of advanced vascular disease, which itself is a risk factor for LEA. What drives the increased risk of LEA with canagliflozin needs to be explored. A possible mechanism may be hemoconcentration due to osmotic diuresis as profound diuresis was observed more often with canagliflozin than placebo $[34.5$ vs 13.3 , $p<0.001$ (event rate/1000 patient-year)] and more severe volume depletion with canagliflozin than empagliflozin (HR 1.44 vs 0.99 with canagliflozin and empagliflozin, respectively) $[3,4]$.

Another eye-opener was a $23 \%$ increased risk for low-trauma fracture (predominantly in upper limb and ribs) with canagliflozin (HR 1.23, 95\% CI 0.99-1.52) [4]. The fracture rates were reported to be similar in both empagliflozin and placebo arms (3.8\% versus 3.9\%), though the fractures were not systematically captured in the EMPA-REG trial [20]. Further, the fractures observed in the two studies were independent of baseline bone mineral density (BMD). The possible explanations for increased risk of fracture risk include increase in serum phosphate and consequent secondary hyperparathyroidism, postural fall due to volume depletion (HR 1.03 with empagliflozin and HR 1.44 with canagliflozin) [21], decrease in estrogen secretion subsequent to weight loss (predominantly fat), and possible decrease in BMD [22]. However, both EMPA-REG and CANVAS were not powerful enough to detect significant differences in either amputation or fracture among the studied population.

\section{CONCLUSIONS}

Both EMPA-REG and CANVAS have provided us an opportunity to tunnel away from the glucocentric approach and think beyond the glycemic benefits of OADs towards improved cardiovascular and renal outcomes. Nevertheless, the comparison of results between 
EMA-REG and CANVAS is confounded by the differences in the enrolled subjects, design of the trials, and analytical approach. Superiority for $\mathrm{CV}$ outcomes is shown by both trials in subjects with prior CVD, but empagliflozin fared better in Asian subjects. Similarly, both trials also showed renoprotective benefits. Risk of amputation and fractures with SGLT2i requires further data to substantiate these observations.

\section{ACKNOWLEDGEMENTS}

No funding or sponsorship was received for this study or publication of this article. The article processing charges were funded by the authors. We appreciate Vikram S. Shekhawat collecting the required reference articles. All named authors meet the International Committee of Medical Journal Editors (ICMJE) criteria for authorship for this manuscript, take responsibility for the integrity of the work as a whole, and have given final approval for the version to be published. Ashu Rastogi and Anil Bhansali contributed equally in literature search, data collection, data interpretation, and writing and editing of the manuscript.

Disclosures. Both Ashu Rastogi and Anil Bhansali have nothing to disclose.

Compliance with Ethics Guidelines. This article is based on previously conducted studies and does not involve any new studies of human or animal subjects performed by any of the authors.

Data Availability. Data sharing is not applicable to this article as no datasets were generated or analyzed during the current study.

Open Access. This article is distributed under the terms of the Creative Commons Attribution-NonCommercial 4.0 International License (http://creativecommons.org/licenses/ by-nc/4.0/), which permits any noncommercial use, distribution, and reproduction in any medium, provided you give appropriate credit to the original author(s) and the source, provide a link to the Creative Commons license, and indicate if changes were made.

\section{REFERENCES}

1. FDA. Guidance for industry: diabetes mellitusevaluating cardiovascular risk in new antidiabetic therapies to treat type 2 diabetes. Washington, DC: US Department of Health and Human Resources; 2008.

2. Schnell O, Ryden L, Standl E, Ceriello A. Current perspectives on cardiovascular outcome trials in diabetes. Cardiovasc Diabetol. 2016;15:139.

3. Zinman B, Wanner C, Lachin JM, et al. Empagliflozin, cardiovascular outcomes, and mortality in type 2 diabetes. N Engl J Med. 2015;373:2117-28.

4. Neal B, Perkovic V, Mahaffey KW, et al. Canagliflozin and cardiovascular and renal events in type 2 diabetes. N Engl J Med. 2017;377:644-57.

5. Holman RR, Paul SK, Bethel A, Mathews DR, Neil AW. 10-year follow-up of intensive glucose control in type 2 diabetes. N Engl J Med. 2008;359:1577-89.

6. Marso MP, Danields GH, Brown-Frandsen K, et al. Liraglutide and cardiovascular outcomes in type 2 diabetes. N Engl J Med. 2016;375:311-22.

7. Neal B, Perkovic V, Mahaffey KW, et al. Rationale, design, and baseline characteristics of the canagliflozin cardiovascular assessment study (CANVAS)a randomized placebo-controlled trial. Am Heart J. 2013;166:217-23.

8. Neal B, Perkovic V, Mahaffey KW, et al. Optimizing the analysis strategy for the CANVAS Program: a prespecified plan for the integrated analyses of the CANVAS and CANVAS-R trials. Diabetes Obes Metab. 2017;19:926-35.

9. Whiting DR, Guariguata L, Weil C, Shaw J. IDF atlas: global estimates of the prevalence of diabetes for 2011 and 2030. Diabetes Res Clin Pract. 2011;94:311-21.

10. Alzaid A. Empa's new clothes: the untold story of the EMPA-REG outcome trial. Diabetes Technol Ther. 2017;19:324-7.

11. Ferranini E, Solini A. SGLT2 inhibitors in diabetes mellitus: rationale and clinical prospects. Nat Rev Endocrinol. 2012;8:495-502. 
12. Abdul-Ghani MA, Norton L, DeFronzo RA. Role of sodium-glucose cotransporters 2 (SGLT2) inhibitors in the treatment of type 2 diabetes. Endocr Rev. 2011;32:515-31.

13. Hirshberg B, Katz A. Insights from cardiovascular outcome trials with novel anti-diabetic agents: what have we learned? An industry perspective. Curr Diabetes Rep. 2015;15:87.

14. McMurray J. EMPA-REG-the "diuretic hypothesis". J Diabetes Complications. 2015;30:3-4.

15. Ferrannini E, Mark M, Mayoux E. CV protection in the EMPA-REG OUTCOME trial: a "Thrifty Substrate" hypothesis. Diabetes Care. 2016;39:1108-14.

16. Mudaliar S, Alloju S, Henry RR. Can a shift in fuel energetics explain the beneficial cardiorenal outcomes in the EMPA-REG OUTCOME study? A unifying hypothesis. Diabetes Care. 2016;39:1115-22.

17. Sardar P, Udell JA, Chatterjee S, Bansilal B, Mukherjee D, Farkouh ME. Effect of intensive versus standard blood glucose control in patients with type 2 diabetes mellitus in different regions of the world: systematic review and meta analysis of randomized controlled trials. JAHA. 2015. doi:10. 1161/JAHA.114.001577.

18. Wanner C, Inzucchi SE, Lachin JM, et al. Empagliflozin and progression of kidney disease in type 2 diabetes. N Engl J Med. 2016;375:323-34.

19. Fadini GP, Avogaro A. SGLT2 inhibitors and amputations in the US FDA adverse events reporting system. Lancet Diabetes Endocrinol. 2017;5:680-1.

20. European Medicines Agency (2014) Summary of the risk management plan (RMP) for Jardiance (empagliflozin). http://www.fimea.fi/download/27456_ Jardiance_RMP_summary-EN.pdf. Accessed 26 Jun 2017.

21. Watts NB, Bilezikian JP, Usiskin $\mathrm{K}$, et al. Effects of canagliflozin on fracture risk in patients with type 2 diabetes mellitus. J Clin Endocrinol Metab. 2016;101:157-66.

22. Bilezikian JP, Atts NB, Usiskin K, et al. Evaluation of bone mineral density and bone markers in patients with type 2 diabetes mellitus treated with canagliflozin, a sodium glucose co-transporter 2 inhibitors. J Clin Endocrinol Metab. 2016;101:44-51. 